\title{
The effects of obesity and type 2 diabetes mellitus on cardiac structure and function in adolescents and young adults
}

\author{
A. S. Shah • P. R. Khoury • L. M. Dolan • \\ H. M. Ippisch • E. M. Urbina • S. R. Daniels • \\ T. R. Kimball
}

Received: 23 September 2010 / Accepted: 22 October 2010 /Published online: 18 November 2010

(C) Springer-Verlag 2010

\begin{abstract}
Aims/hypothesis We sought to evaluate the effects of obesity and obesity-related type 2 diabetes mellitus on cardiac geometry (remodelling) and systolic and diastolic function in adolescents and young adults.

Methods Cardiac structure and function were compared by echocardiography in participants who were lean, obese or obese with type 2 diabetes (obese diabetic), in a cross sectional study. Group differences were assessed using ANOVA. Independent determinants of cardiac outcome measures were evaluated with general linear models.

Results Adolescents with obesity and obesity-related type 2 diabetes were found to have abnormal cardiac geometry compared with lean controls $(16 \%$ and $20 \%$ vs $<1 \%$, $p<0.05)$. These two groups also had increased systolic function. Diastolic function decreased from the lean to obese to obese diabetic groups with the lowest diastolic function observed in the obese diabetic group $(p<0.05)$. Regression analysis showed that group, BMI $z$ score $(\mathrm{BMIz})$, group $\times$ BMIz interaction and systolic BP $z$ score
\end{abstract}

A. S. Shah $(\bowtie) \cdot$ L. M. Dolan

Cincinnati Children's Hospital Medical Center,

Division of Endocrinology,

3333 Burnet Avenue, MLC 7012,

Cincinnati, OH 45229, USA

e-mail: amy.shah@cchmc.org

P. R. Khoury $\cdot$ H. M. Ippisch • E. M. Urbina • T. R. Kimball

Cincinnati Children's Hospital Medical Center,

Division of Cardiology,

Cincinnati, OH, USA

S. R. Daniels

University of Colorado Denver School of Medicine,

Denver, CO, USA
(BPz) were significant determinants of cardiac structure, while group, BMIz, systolic BPz, age and fasting glucose were significant determinants of the diastolic function (all $p<0.05$ ).

Conclusions/interpretation Adolescents with obesity and obesity-related type 2 diabetes demonstrate changes in cardiac geometry consistent with cardiac remodelling. These two groups also demonstrate decreased diastolic function compared with lean controls, with the greatest decrease observed in those with type 2 diabetes. Adults with diastolic dysfunction are known to be at increased risk of progressing to heart failure. Therefore, our findings suggest that adolescents with obesity-related type 2 diabetes may be at increased risk of progressing to early heart failure compared with their obese and lean counterparts.

Keywords Cardiac geometry - Diastolic function - Obesity . Systolic function - Type 2 diabetes

\begin{tabular}{ll}
\multicolumn{2}{l}{ Abbreviations } \\
A & Late LV filling \\
Aa & Late annular flow \\
ANCOVA & Analysis of covariance \\
BMIz & BMI $z$ score \\
BPz & BP $z$ score \\
CDC-NCS & Centers for Disease Control and Prevention \\
& National Centers for Health Statistics \\
E & Early LV filling \\
Ea & Early annular flow \\
LV & Left ventricular \\
RWT & Relative wall thickness \\
VCF & Velocity of circumferential fibre shortening
\end{tabular}


Adults with obesity or obesity-related type 2 diabetes mellitus are at increased risk for the development of early cardiovascular disease [1]. Both obesity and type 2 diabetes are known to cause deleterious abnormalities in cardiac structure and function independent of coronary artery disease and hypertension [2]. Specifically, these processes lead to abnormal cardiac geometry (remodelling) and diastolic dysfunction [3-6]. In fact, several studies have indicated that left ventricular (LV) diastolic dysfunction represents the earliest preclinical manifestation of diabetic cardiomyopathy, which can progress to symptomatic heart failure $[7,8]$.

Given the findings in adults, we hypothesise that adolescents and young adults with obesity and obesityrelated type 2 diabetes will demonstrate early changes in cardiac geometry and diastolic function, suggesting that they are at increased risk for the development of premature heart failure. We also hypothesise that individuals with obesity-related type 2 diabetes will have poorer cardiac structure and function compared with their counterparts with obesity alone.

\section{Methods}

\section{Study population}

Adolescents and young adults were evaluated as part of a cross sectional study examining the role of obesity and type 2 diabetes on cardiac and vascular function in youth. Three study groups were recruited (1) obese participants with type 2 diabetes; (2) obese control participants (i.e. obese patients without type 2 diabetes); and (3) lean control participants (non-obese without type 2 diabetes). The age of the participants was between 10 and 24 years. Pregnant females and those with congenital heart disease were excluded from the study. Obese participants had a BMI $\geq 95$ th percentile while lean participants had a BMI <85th percentile, definitions based on the Centers for Disease Control and Prevention National Centers for Health Statistics (CDCNCHS) growth charts [9]. Diagnosis of type 2 diabetes was based on the ADA criteria [10]. Specifically, participants had either elevated fasting plasma glucose levels $\geq 7.0 \mathrm{mmol} / 1$ $(\geq 126 \mathrm{mg} / \mathrm{dl})$, symptoms of hyperglycaemia and random plasma glucose $\geq 11.1 \mathrm{mmol} / 1(\geq 200 \mathrm{mg} / \mathrm{dl})$ or $2 \mathrm{~h}$ plasma glucose $\geq 11.1 \mathrm{mmol} / 1(\geq 200 \mathrm{mg} / \mathrm{dl})$ during an oral glucose tolerance test. All individuals with type 2 diabetes had no evidence of other specific types of diabetes and were noninsulin requiring in the basal state. A total of 147 participants were islet cell antibody-negative (glutamic acid decarboxylase, islet cell antigen 512, insulin autoantibodies). Ten individuals did not have islet cell antibody data available but met ADA criteria for the diagnosis of type 2 diabetes.
The study population was recruited from clinics at Cincinnati Children's Hospital, community physician offices, city clinics, health fairs, and college campuses. The ten diabetic participants recruited from community physicians did not have islet cell antibody data. Before enrolment in the study, written informed consent was obtained from participants who were $\geq 18$ years old or the parent or guardian for participants who were $<18$ years old, according to the guidelines established by the Institutional Review Board at Cincinnati Children's Hospital Medical Center and in accordance with the Declaration of Helsinki.

\section{Data / laboratory collection}

After a minimum $10 \mathrm{~h}$ overnight fast, participants came to the Clinical Research Center at Cincinnati Children's Hospital Medical Center for an in-person study visit where demographic, laboratory and anthropometric details and blood pressure were taken as previously reported [11]. The BMIz score was calculated for each participant using the CDC-NCHS growth reference data $[9,12]$. Waist circumference was measured to the nearest $0.5 \mathrm{~cm}$ using a flexible metal research tape twice and averaged according to standardised research protocol from the National Heart, Lung and Blood Institute Health Study. Dual energy x-ray absorptiometry (Hologic 4500A, Bedford, MA, USA) was performed to determine lean mass and per cent body fat. Per cent body fat was calculated as total body fat mass divided by total body mass multiplied by 100 . Glycosylated haemoglobin $\left(\mathrm{HbA}_{1 \mathrm{c}}\right)$ was measured in erythrocytes using high performance liquid chromatography methods. Duration of type 2 diabetes was calculated from the date of diagnosis to the date of study.

\section{Echocardiography}

The heart was non-invasively assessed with a GE Vivid 5 or 7 (Milwaukee, WI, USA) or Philips Sonos 5500 (Andover, MA, USA) ultrasound system. A complete two-dimensional pulsed Doppler, tissue Doppler and colour Doppler echocardiography examination was performed on each participant. All images were obtained with the participant in the left decubitus position to acquire parasternal long and short axis and apical four chamber views. A total of three cardiac cycles were measured for each participant by a sonographer blinded to group. Measurements were performed off-line by a single technician blinded to group using a Cardiology Analysis System (Digisonics, Houston, TX, USA).

Echocardiography measurements

Cardiac structure and geometry LV mass index mass (a measure of hypertrophy) and relative wall thickness 
(RWT) (a measure of LV wall thickness relative to $\mathrm{LV}$ cavity size) were calculated using indices of LV enddiastolic dimension, end-diastolic posterior wall thickness and end-diastolic septal thickness as previously described [13]. LV mass was indexed by dividing LV mass by height in meters raised to 2.7 to minimise the effects of age, sex and race $[14,15]$. Threshold levels for LV mass index and RWT were used to evaluate LV geometry. For LV mass index, an adult threshold of $>51 \mathrm{~g} / \mathrm{m}^{2.7}$ was used because of its association with cardiac morbidity in adults [16]. For RWT, a common adult threshold of $>0.43$ was used $[4,17]$. This resulted in four geometric categories: normal geometry, concentric remodelling (normal LV mass index and increased RWT), eccentric hypertrophy (increased LV mass index and normal RWT), and concentric hypertrophy (increased LV mass index and increased RWT). These categories of LV geometry are related to cardiovascular mortality, with concentric hypertrophy being the most severe, and are often a precursor to LV failure [18].

Cardiac function LV systolic function was assessed by measures previously described [13]. Systolic outcome measures included shortening fraction, end-systolic wall stress and velocity of circumferential fibre shortening difference. Shortening fraction is a measure of LV systolic performance and wall stress is a measure of cardiac afterload calculated from end-systolic dimensions and pressure. Velocity of circumferential fibre shortening (VCF) difference is a measure of contractility, calculated as measured VCF less predicted VCF for each participant's given wall stress.

More than one measure was also used to evaluate diastolic function. First, Doppler interrogation of transmitral blood flow velocities was used to measure peak early (E) and late (A) LV filling. The ratio of both was used to calculate E/A. Tissue Doppler analyses of the myocardial wall velocities at both the septal and lateral annuli was also performed to obtain peak early (Ea) and late (Aa) velocities of mitral annular flow. The ratios of these measures at the septal and lateral annuli were averaged to calculate $\mathrm{Ea} / \mathrm{Aa}$ average. Finally, the ratio of mitral to myocardial early filling was measured to calculate $\mathrm{E} / \mathrm{Ea}$ average, a measure of LV end-diastolic pressure [19]. These particular noninvasive measures of diastolic function correlate well with invasive measures of diastolic function (time constant of relaxation [tau]) and LV end-diastolic pressure [20].

Statistical analysis

All analyses were performed with Statistical Analysis Software (SAS, version 9.1.3) [21]. Average values for demographic, anthropometric, blood pressure and laboratory values were obtained for each group. Non-normally distributed variables were $\log$ transformed to the $\log _{\mathrm{e}}$ for comparison. ANOVA was performed to compare means among groups using Bonferroni correction methods for multiple comparisons. Differences in LV geometry among groups were assessed by $\chi^{2}$ analysis. Bivariate correlations were calculated between LV mass index (component of cardiac geometry), systolic (shortening fraction) and diastolic outcomes (E/A, Ea/Aa average, E/Ea average) and potential demographic and physiologic predictors. HbAlc, glucose and duration of disease were only considered for participants with type 2 diabetes. Significant variables from the correlation analyses were then used to construct analysis of covariance (ANCOVA) models using proc GLM for each outcome variable. The full model potentially included: demographic (group, age, race, sex), anthropometric (BMIz, waist circumference, lean mass from dualenergy x-ray absorptiometry [DXA]), and haemodynamic (systolic and diastolic BPz scores) variables. RWT and LV mass indices were only included in the models constructed for diastolic variables. Group was forced into the model as a class variable to ensure group differences would be considered when determining significant predictors. The significance level of each covariate was assessed with the most significant term added in a stepwise manner. Any nonsignificant term was removed after each step. This process continued until all remaining covariates were significant. Interaction terms between the group and the independent predictors were included in the models and tested for significance. Any non-significant interaction term was also removed from the final models.

\section{Results}

The study population consisted of 612 adolescents. Table 1 lists the mean and standard deviations for anthropometric, blood pressure and laboratory data for all participants stratified by group (lean, obese, obese diabetic). The groups did not differ by age or sex distribution $(p<0.05)$. As dictated by study design, lean individuals had a lower BMI than obese and obese diabetic participants. Cardiovascular risk profile (weight, waist circumference and systolic blood pressure) worsened across groups from lean to obese to obese diabetic $(p<0.05)$. As expected, participants with type 2 diabetes had a significantly higher fasting glucose and $\mathrm{HbA} 1 \mathrm{c}$ compared with lean and obese groups. Average duration of disease in diabetic participants was $3.2 \pm$ 2.5 years.

The absolute number of participants with abnormal BP levels was lower in the lean $(13.4 \%$ prehypertensive, $<0.01 \%$ with stage 1 or stage 2 hypertension) and obese groups $(30.5 \%$ prehypertensive, $5.8 \%$ with stage 1 or stage 2 hypertension), with the obese diabetic group having 
Table 1 Demographics and body composition of the study population by group
Values are mean $\pm \mathrm{SD}$

${ }^{\mathrm{a}}$ Lean and obese $<$ obese diabetic, $p<0.05$

${ }^{\mathrm{b}}$ Lean $<$ obese $<$ obese diabetic, $p<0.05$

${ }^{\mathrm{c}}$ Lean $<$ obese and obese diabetic, $p<0.05$

\begin{tabular}{|c|c|c|c|}
\hline Variable & Lean & Obese & Obese diabetic \\
\hline$n$ & 232 & 223 & 157 \\
\hline Age (years) & $17.8 \pm 3.5$ & $18.1 \pm 3.2$ & $17.9 \pm 3.2$ \\
\hline Age range (years) & $10.1-24$ & $10.4-24$ & $10.2-24$ \\
\hline Female sex $(\%)$ & $143(62)$ & $158(71)$ & $103(66)$ \\
\hline Race (white) (\%) & $106(46)$ & $69(31)$ & $68(43)$ \\
\hline Race (black) (\%) & $122(53)$ & $153(69)$ & $83(53)$ \\
\hline Race (other/mixed race) $(\%)$ & $4(1)$ & $1(<1)$ & $6(4)$ \\
\hline Height $(\mathrm{cm})^{a}$ & $165.5 \pm 11.0$ & $166.4 \pm 9.8$ & $169.1 \pm 10.6$ \\
\hline Weight $(\mathrm{kg})^{\mathrm{b}}$ & $58.8 \pm 11.7$ & $104.4 \pm 20.1$ & $112.3 \pm 24.2$ \\
\hline BMI $\left(\mathrm{kg} / \mathrm{m}^{2}\right)^{\mathrm{b}}$ & $21.3 \pm 2.4$ & $37.6 \pm 6.9$ & $39.1 \pm 7.1$ \\
\hline Lean mass $(\mathrm{kg})^{\mathrm{b}}$ & $43.7 \pm 10.5$ & $59.1 \pm 11.2$ & $62.4 \pm 12.3$ \\
\hline Waist circumference $(\mathrm{cm})^{b}$ & $75.9 \pm 7.3$ & $114.7 \pm 15.8$ & $121.3 \pm 15.3$ \\
\hline $\mathrm{SBP}(\mathrm{mmHg})^{\mathrm{b}}$ & $108 \pm 10$ & $117 \pm 11$ & $123 \pm 13$ \\
\hline $\mathrm{DBP}(\mathrm{mmHg})^{\mathrm{c}}$ & $60.0 \pm 12.0$ & $66 \pm 12$ & $67 \pm 14$ \\
\hline Fasting glucose $(\mathrm{mmol} / \mathrm{l})^{a}$ & $4.9 \pm 0.33$ & $5.1 \pm .44$ & $8.2 \pm 4.3$ \\
\hline $\mathrm{HbA}_{1 \mathrm{c}} \%{ }^{\mathrm{a}}$ & $5.3 \pm 0.5$ & $5.5 \pm 0.4$ & $7.8 \pm 2.8$ \\
\hline Duration of diabetes & - & - & $3.2 \pm 2.5$ years \\
\hline
\end{tabular}

higher prevalence of hypertension (46\% prehypertensive, $16.6 \%$ with stage 1 or 2 hypertension).

The presence of dyslipidaemia was also assessed. Dyslipidaemia was defined by LDL $>3.4 \mathrm{mmol} / \mathrm{l}$, TG $>14.5 \mathrm{mmol} / \mathrm{l}$ or HDL $<1.0 \mathrm{mmol} / \mathrm{l}$ [22-24]. In the lean group $5.2 \%$ of participants were found to have evidence of dyslipidaemia, which was significantly lower than those in the obese and (24.2\%) and obese diabetic group (26.2\%). Review of medication history revealed that three participants in the obese group were taking metformin, while in the obese diabetic group 86 were taking metformin and one was taking long-acting insulin glargine (A21Gly,B31Arg, B32Arg human insulin). BP medication history revealed two obese participants and 27 diabetic participants were taking anti-hypertensive medications.

Measures of cardiac structure and geometry by group are listed in Table 2. Compared with the lean controls, LV mass index was increased but similar in both the obese and obese diabetic groups $(p<0.05)$. Further analysis of the components of LV mass index demonstrated that increased LV mass index was a result of increased LV end-diastolic dimension and septal and posterior wall thickness in the heart. RWT was also higher in the obese and obese diabetic participants compared with lean participants, but average RWT did not exceed threshold levels that would contribute to abnormal geometry. The presence of any type of abnormal geometry was similar for the obese and obese diabetic group (16\% vs $20 \%$ ), both higher than for the lean group, who had abnormal geometry less than $1 \%$ of the time $(p<0.05)$. The most prevalent abnormal geometry type in both the obese and obese diabetic groups was eccentric hypertrophy (increased LV mass index, normal RWT).
LV systolic function measures are shown in Table 2. The shortening fraction was higher in both obese and obese diabetic participants compared with lean controls but it was not different from one another, indicating a similar increase in LV performance. Wall stress was lower in the obese and obese diabetic groups compared with the lean group. There were no significant differences in VCF difference (contractility) among the three groups.

Diastolic function differed among the three groups (see Fig. 1). Compared with the lean group, diastolic function was decreased in the obese group and further decreased in the obese diabetic group. Specifically, obese participants had a higher mitral E/Ea average (increased LV filling pressure) and lower mitral Ea/Aa average (decreased diastolic function) compared with lean participants. The obese diabetic group had the highest E/Ea average and lowest $\mathrm{Ea} / \mathrm{Aa}$ average. In this group, E/A ratio was also lower compared with obese and lean controls (decreased diastolic function) (Table 2). Five individuals in the obese diabetic group had E/Ea mean $>10$, suggesting abnormal diastolic filling pressure $[25,26]$. There were no lean or obese non-diabetic patients who met this criterion.

Table 3 demonstrates significant correlation coefficients for the LV mass index, shortening fraction and diastolic function outcomes. Age, adiposity (BMIz, waist circumference and per cent body fat), lean mass, and $\mathrm{BPz}$ were significantly associated with LV mass index. Only adiposity and systolic $\mathrm{BPz}$ were significantly associated with the shortening fraction. Waist circumference, systolic $\mathrm{BPz}$ and LV mass index were significantly correlated with all three measures of diastolic function. Type 2 diabetes measures (fasting glucose, $\mathrm{HbA}_{1 \mathrm{c}}$ and duration of diabetes) were 
Table 2 Echocardiography measurements of the population by group
Values are mean $\pm \mathrm{SD}$

${ }^{a}$ Lean vs obese and obese diabetic, $p<0.05$

${ }^{\mathrm{b}}$ Lean vs obese vs obese diabetic, $p<0.05$

${ }^{\mathrm{c}}$ Lean and obese vs obese diabetic, $p<0.05$

avg, average

\begin{tabular}{|c|c|c|c|}
\hline $\begin{array}{l}\text { Variable } \\
n\end{array}$ & $\begin{array}{l}\text { Lean } \\
232\end{array}$ & $\begin{array}{l}\text { Obese } \\
223\end{array}$ & $\begin{array}{l}\text { Obese diabetic } \\
157\end{array}$ \\
\hline \multicolumn{4}{|l|}{ Cardiac structure } \\
\hline LV mass index ${ }^{2.7}(\mathrm{~g} / \mathrm{m})^{\mathrm{a}}$ & $27.3 \pm 6.1$ & $39.0 \pm 9.3$ & $40.4 \pm 9.8$ \\
\hline LV end-diastolic dimension $(\mathrm{cm})^{\mathrm{a}}$ & $4.84 \pm 0.48$ & $5.28 \pm 0.51$ & $5.25 \pm 0.59$ \\
\hline LV posterior wall thickness-diastolic $(\mathrm{cm})^{\mathrm{a}}$ & $0.67 \pm 0.12$ & $0.82 \pm 0.14$ & $0.87 \pm 0.15$ \\
\hline End-diastolic septal thickness $(\mathrm{cm})^{\mathrm{a}}$ & $0.69 \pm 0.13$ & $0.81 \pm 0.16$ & $0.85 \pm 0.16$ \\
\hline $\mathrm{RWT}^{\mathrm{a}}$ & $0.28 \pm 0.05$ & $0.31 \pm 0.06$ & $0.33 \pm 0.07$ \\
\hline \multicolumn{4}{|l|}{ Geometry $n(\%)$} \\
\hline Normal & $230(99)$ & $187(83.8)$ & $125(79.6)$ \\
\hline Concentric remodelling & $1(<0.01)$ & $8(3.6)$ & $10(6.4)$ \\
\hline Eccentric hypertrophy & 0 & $26(11.7)$ & $17(10.8)$ \\
\hline Concentric hypertrophy & $1(<0.01)$ & $2(<0.01)$ & $5(3.2)$ \\
\hline \multicolumn{4}{|l|}{ Systolic function } \\
\hline Shortening fraction $(\%)^{\mathrm{a}}$ & $36.2 \pm 4.0$ & $37.4 \pm 4.1$ & $37.4 \pm 4.3$ \\
\hline Wall stress $\left(\mathrm{g} / \mathrm{cm}^{2}\right)^{\mathrm{a}}$ & $54.9 \pm 13.7$ & $50.1 \pm 14.4$ & $49.1 \pm 14.2$ \\
\hline Systolic pressure $(\mathrm{mmHg})^{\mathrm{a}}$ & $83.49 \pm 10.41$ & $89.05 \pm 12.40$ & $91.17 \pm 11.71$ \\
\hline $\mathrm{LV}$ end-systolic dimension $(\mathrm{cm})^{\mathrm{a}}$ & $3.09 \pm 0.39$ & $3.30 \pm 0.41$ & $3.29 \pm 0.44$ \\
\hline LV posterior wall thickness-systolic $(\mathrm{cm})^{\mathrm{a}}$ & $1.18 \pm 0.17$ & $1.43 \pm 0.23$ & $1.50 \pm 0.23$ \\
\hline VCF difference (circ/s) & $0.04 \pm 0.29$ & $0.06 \pm 0.28$ & $0.05 \pm 0.25$ \\
\hline \multicolumn{4}{|l|}{ Diastolic function } \\
\hline Left atrial diameter $(\mathrm{cm})^{\mathrm{a}}$ & $2.92 \pm 0.41$ & $3.55 \pm 0.45$ & $3.63 \pm 0.48$ \\
\hline Left atrial volume $(\mathrm{ml})^{\mathrm{a}}$ & $25.2 \pm 9.8$ & $29.9 \pm 9.4$ & $30.0 \pm 10.9$ \\
\hline Peak $E^{a}$ & $90.1 \pm 14.5$ & $98.2 \pm 17.3$ & $98.8 \pm 17.1$ \\
\hline Peak $A^{b}$ & $46.6 \pm 11.4$ & $51.1 \pm 11.2$ & $58.1 \pm 12.3$ \\
\hline Mitral E/A ratio ${ }^{\mathrm{c}}$ & $2.02 \pm 0.53$ & $2.03 \pm 0.59$ & $1.79 \pm 0.44$ \\
\hline Mitral Ea/Aa-avg ${ }^{\mathrm{b}}$ & $2.63 \pm 0.64$ & $2.20 \pm 0.59$ & $2.04 \pm 0.55$ \\
\hline Mitral E/Ea-avg ${ }^{\mathrm{b}}$ & $5.74 \pm 1.17$ & $6.77 \pm 1.30$ & $7.31 \pm 1.53$ \\
\hline Mitral E/Ea-avg $>10$ & 0 & 0 & 5 \\
\hline
\end{tabular}

significantly associated with LV mass index, Ea/Aa average and $\mathrm{E} / \mathrm{Ea}$ average.

The ANCOVA models are presented in Table 4. A model for shortening fraction was constructed but omitted from the manuscript as the amount of variation explained by the model was too low to be considered meaningful. Group

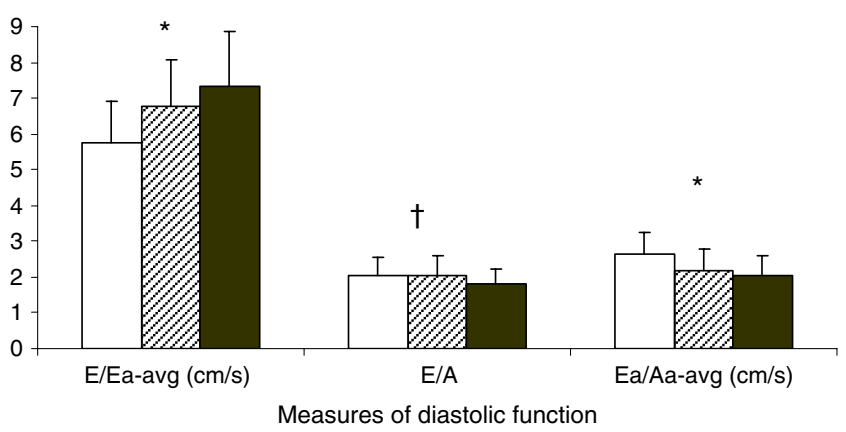

Fig. 1 Diastolic function by group (mean and SD). White bars, lean; cross-hatched bars, obese; black bars, type 2 diabetes. Lean $<$ obese $<$ obese type 2 diabetes, ${ }^{*} p<0.05$. Lean and obese $<$ obese type 2 diabetes, ${ }^{\dagger} p<0.05$. avg, average was a significant predictor of $\mathrm{E} / \mathrm{Ea}$, indicating that the mean values of $\mathrm{E} / \mathrm{Ea}$ differed among the three groups, after adjusting for other factors in the model. For LV mass index, there was a significant BMIz by group interaction in the obese diabetic group, indicating the regression slope in this group was significantly higher than in the lean group. Cardiovascular risk factors were also important in explaining the variance in cardiac structure and function. Higher systolic $\mathrm{BPz}$ and waist circumference were associated with higher LV mass index and poorer diastolic function. Additionally, higher fasting glucose was associated with poorer diastolic function but did not contribute to increased LV mass index (for all model and variable estimates $p<0.05)$. Figure 2 is presented to visually demonstrate the differences in LV mass index and $\mathrm{BMIz}$ for study design groups described in Table 4 . The slope of the regression line is different among all three groups with the obese diabetic group having the steepest slope. This suggests that there is an additional burden on the heart in the obese diabetic group beyond what is expected from obesity alone. 
Table 3 Bivariate correlations ( $r$ values) of LV mass index and systolic and diastolic variables

Significant $(p<0.01) r$ values unless otherwise indicated by NS (non-significant)

Fasting glucose, $\mathrm{HbAlc}$ and Duration of T2DM only considered for the obese diabetic group

Avg, average

\begin{tabular}{llllll}
\hline Variable & LV mass index & Shortening fraction & E/A & Ea/Aa avg & E/Ea avg \\
\hline Age & 0.143 & NS & -0.129 & -0.179 & NS \\
BMIz & 0.639 & 0.125 & NS & -0.379 & 0.427 \\
\% Body fat & 0.453 & 0.211 & NS & -0.283 & 0.367 \\
Lean mass & 0.537 & NS & NS & -0.324 & 0.236 \\
Waist circumference & 0.644 & 0.122 & -0.163 & -0.398 & 0.409 \\
Systolic BPz & 0.440 & 0.136 & -0.130 & -0.327 & 0.323 \\
Diastolic BPz & 0.121 & NS & NS & -0.118 & 0.167 \\
LV mass index & 1.00 & $\mathrm{NS}$ & -0.131 & -0.247 & 0.336 \\
Fasting glucose & 0.172 & $\mathrm{NS}$ & $\mathrm{NS}$ & -0.226 & 0.190 \\
HbA & 0.138 & $\mathrm{NS}$ & -0.127 & -0.232 & 0.227 \\
Duration of diabetes & -0.157 & $\mathrm{NS}$ & $\mathrm{NS}$ & -0.215 & $\mathrm{NS}$ \\
\hline
\end{tabular}

\section{Discussion}

This study documents that adolescents and young adults with obesity and obesity-related type 2 diabetes have a higher frequency of abnormal cardiac geometry, increased systolic function and decreased diastolic function compared with age-matched lean controls. These data also demonstrate that obese adolescents with type 2 diabetes have a further decline in diastolic function compared with nondiabetic obese adolescents. In adults, diastolic dysfunction is a significant risk factor for future development of congestive heart failure $[8,27]$; therefore, our data suggest that obese adolescents with type 2 diabetes may be at higher risk of progressing to premature heart failure compared with their non-diabetic obese and lean counterparts.

Adults with obesity and obesity-related type 2 diabetes demonstrate increased LV mass index and increased RWT leading to a predominant cardiac geometry of concentric hypertrophy [5, 6, 28-31]. To date, no study has compared cardiac geometry in lean, obese and obese diabetic adolescents. Our data demonstrate that, compared with lean controls, adolescents and young adults with obesity and obesity-related type 2 diabetes have an increased LV mass index, contributing to a higher frequency of eccentric hypertrophy (increased LV mass and normal RWT). Although RWT was higher in obese and obese diabetic participants compared with lean controls, their RWT values did not exceed threshold levels to contribute to abnormal geometry (RWT >0.43). These findings suggest that an increase in RWT may be a late phenomenon and is likely to be a result of sustained increased haemodynamic load on the heart. As a result, adolescents with obesity and obesityrelated type 2 diabetes may be at risk of progressing to concentric hypertrophy, the cardiac geometry commonly

Table 4 Regression coefficients from ANCOVA on LV mass index and diastolic variables

\begin{tabular}{|c|c|c|c|c|c|}
\hline Variable & Group & LV mass index & $\mathrm{E} / \mathrm{A}$ & $\mathrm{Ea} / \mathrm{Aa}$ avg & E/Ea avg \\
\hline Intercept & & 3.10 & 1.81 & 1.68 & 1.89 \\
\hline \multirow[t]{3}{*}{ Group } & Lean & (reference group) & & (reference group) & (reference group) \\
\hline & Obese & $-0.118+$ & -0.048 & $-0.066+$ & 0.0131 \\
\hline & Obese diabetic & -0.288 & $-0.066+$ & $-0.094+$ & 0.203 \\
\hline BMIz & & 0.075 & & & 0.050 \\
\hline \multirow[t]{3}{*}{ Group $\times$ BMIz interaction } & Lean & 0 (reference group) & & & \\
\hline & Obese & $0.093+$ & & & \\
\hline & Obese diabetic & 0.155 & & & \\
\hline Lean mass & & 0.004 & & & \\
\hline Systolic BPz & & 0.051 & -0.038 & -0.034 & 0.044 \\
\hline Glucose & & & -0.132 & -0.106 & \\
\hline Waist circumference & & & -0.002 & -0.002 & \\
\hline Age & & & -0.007 & -0.008 & -0.008 \\
\hline$R^{2}$ & & 0.50 & 0.22 & 0.22 & 0.25 \\
\hline
\end{tabular}

All variable estimates are $p<0.05$

Group forced in the model. If not significant, denoted by + 


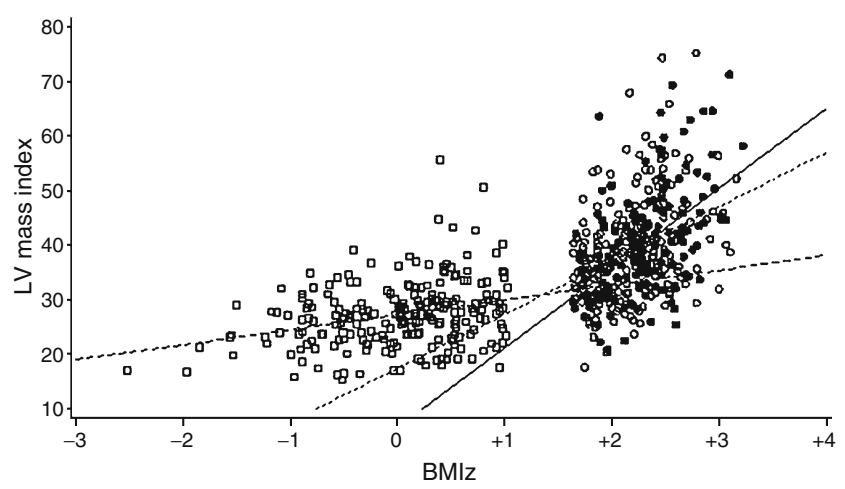

Fig. 2 Regression of LVM index on BMIz. White squares/long dashed line, lean; white circles/short dashed line, obese; black square/ continuous line, obese type 2 diabetes

found in obese adults and adults with type 2 diabetes. This possibility has been suggested in work published by Ippisch et al., in which concentric hypertrophy was the predominant cardiac geometric pattern in adolescents with morbid obesity [13].

It is normal for systolic function to increase in adults early in the course of obesity and diabetes. There appears to be a compensatory increase in ejection fraction and shortening fraction $[3,30,32]$, which declines with the duration of disease [3] and as it develops in severity [30]. In adolescents there is a paucity of data regarding the effects of obesity and obesity-related type 2 diabetes on systolic function. Ippisch et al. studied morbidly obese adolescents and described increased systolic function (as measured by stroke volume and cardiac output), which was unchanged with weight loss after bariatric surgery [13]. In type 1 diabetes a similar increase in systolic function has been described [33].

Our data show that young individuals with obesity and obesity-related T2DM have increased systolic function as measured by increased shortening fraction and lower wall stress. The changes in wall stress appear to be a result of increased systolic pressure, increased LV ventricular endsystolic dimension and even higher posterior wall thickness in obese and obese diabetic individuals. Similarly to adults, this appears to be a compensatory increase in systolic function. The significance of this increase in systolic function early in the course of both type 1 and type 2 diabetes in adolescents is not clear. Longitudinal studies are needed in this population to determine if systolic function declines with the duration and severity of disease.

Published reports document diastolic dysfunction in obese adults, both with and without type 2 diabetes. Both the duration of disease [3] and severity [30] of obesity and obesity-related type 2 diabetes appear to influence the extent of diastolic dysfunction. Changes are seen in as little as $4-5$ years [34]. In adults with type 2 diabetes, the prevalence of diastolic dysfunction is between $30 \%$ and
$50 \%[34,35]$. In adolescents, reports of the effects of obesity and type 2 diabetes on diastolic function have yielded discrepant results. Specifically, the Strong Heart Study demonstrated no differences in diastolic function (E/A ratio) between obese and lean controls [36]. In contrast, Sharpe et al. demonstrated a decrease in E/A in obese, compared with lean adolescents [37]. Only one previous study has investigated the relationship between type 2 diabetes and diastolic function in adolescents; Whalley et al demonstrated a decreased E/A ratio and increased $\mathrm{E} / \mathrm{Ea}$ ratio early in the course of type 2 diabetes in female adolescents compared with obese and lean controls [38].

Similarly to the above studies, we demonstrate decreased diastolic function in obese adolescents compared with lean controls, and an even further decrease in diastolic function in obese adolescents with type 2 diabetes. We extend previous observations in the literature by studying both males and females. Additionally, from our general linear models, we provide data that demonstrate that this decrease in diastolic function in obese diabetic adolescents is probably due to independent contributions made by increased systolic blood pressure, adiposity and increased fasting glucose. These findings suggest that factors unique to type 2 diabetes (increased fasting glucose) may contribute to the difference in diastolic dysfunction between obese and obese diabetic individuals.

\section{Limitations}

This study has limitations. First, our cross sectional design does not allow us to determine the time sequence for the development of changes in cardiac structure and function as individuals move from lean to obese to type 2 diabetes. Second, unlike trends in longitudinal analysis, we cannot show cause and effect. Third, although we can assess the effect of duration of type 2 diabetes, we cannot assess the duration of obesity in either the obese or obese diabetic group. Finally, we did not study the potential influence of sleep apnoea on cardiac structure and function in this study.

\section{Conclusions}

We conclude that adolescents and young adults with obesity and obesity-related type 2 diabetes have a higher likelihood of abnormal geometry and increased systolic function compared with lean individuals. Furthermore, obese individuals have diastolic dysfunction that declines further in the presence of type 2 diabetes. Since diastolic dysfunction is known to be associated with progression to heart failure in adults $[7,8]$, our findings suggest that obese adolescents 
both with and without type 2 diabetes are at risk for premature heart failure. However, the risk may be greater in obese adolescents with type 2 diabetes.

Acknowledgements This study was supported by National Institutes of Health (NIH-HLBI) grant R01-HL076269 (Cardiovascular Disease in Adolescents with Type 2 Diabetes) and in part by USPHS Grant \#UL1 RR026314 (National Center for Research Resources, NIH). The authors acknowledge the work of V. Moore, C. Schulte, C. McWhorter, T. Bailey and the participants of the Cardiovascular Disease in Type 2 Diabetes Study.

Duality of interest The authors declare that there is no duality of interest associated with this manuscript

\section{References}

1. Ninomiya JK, L'Italien G, Criqui MH, Whyte JL, Gamst A, Chen RS (2004) Association of the metabolic syndrome with history of myocardial infarction and stroke in the Third National Health and Nutrition Examination Survey. Circulation 109:42-46

2. Factor SM, Minase T, Sonnenblick EH (1980) Clinical and morphological features of human hypertensive-diabetic cardiomyopathy. Am Heart J 99:446-458

3. Alpert MA, Lambert CR, Panayiotou H et al (1995) Relation of duration of morbid obesity to left ventricular mass, systolic function, and diastolic filling, and effect of weight loss. Am J Cardiol 76:1194-1197

4. de Simone G, Daniels SR, Kimball TR et al (2005) Evaluation of concentric left ventricular geometry in humans: evidence for agerelated systematic underestimation. Hypertension 45:64-68

5. Devereux RB, Roman MJ, Paranicas M et al (2000) Impact of diabetes on cardiac structure and function: the strong heart study. Circulation 101:2271-2276

6. Lauer MS, Anderson KM, Kannel WB, Levy D (1991) The impact of obesity on left ventricular mass and geometry. The Framingham Heart Study. JAMA 266:231-236

7. Beljic T, Miric M (1994) Improved metabolic control does not reverse left ventricular filling abnormalities in newly diagnosed non-insulin-dependent diabetes patients. Acta Diabetol 31:147-150

8. Vanninen E, Mustonen J, Vainio P, Lansimies E, Uusitupa M (1992) Left ventricular function and dimensions in newly diagnosed non-insulin-dependent diabetes mellitus. Am J Cardiol 70:371-378

9. Centers for Disease Control and Prevention. CDC growth charts. Available from www.cdc.gov/growthcharts/cdc charts.htm, accessed 1 June 2009

10. American Diabetes Association (2008) Standards of medical care in diabetes-2008. Diabetes Care 31(Suppl 1):S12-S54

11. Shah AS, Dolan LM, Kimball TR et al (2009) Influence of duration of diabetes, glycemic control, and traditional cardiovascular risk factors on early atherosclerotic vascular changes in adolescents and young adults with type 2 diabetes mellitus. J Clin Endocrinol Metab 94:3740-3745

12. Cole TJ (1990) The LMS method for constructing normalized growth standards. Eur J Clin Nutr 44:45-60

13. Ippisch HM, Inge TH, Daniels SR et al (2008) Reversibility of cardiac abnormalities in morbidly obese adolescents. J Am Coll Cardiol 51:1342-1348

14. Daniels SR, Kimball TR, Morrison JA, Khoury P, Meyer RA (1995) Indexing left ventricular mass to account for differences in body size in children and adolescents without cardiovascular disease. Am J Cardiol 76:699-701

15. de Simone G, Daniels SR, Devereux RB et al (1992) Left ventricular mass and body size in normotensive children and adults: assessment of allometric relations and impact of overweight. J Am Coll Cardiol 20:1251-1260

16. de Simone G, Devereux RB, Daniels SR, Koren MJ, Meyer RA, Laragh JH (1995) Effect of growth on variability of left ventricular mass: assessment of allometric signals in adults and children and their capacity to predict cardiovascular risk. J Am Coll Cardiol 25:1056-1062

17. Ganau A, Saba PS, Roman MJ, de Simone G, Realdi G, Devereux RB (1995) Ageing induces left ventricular concentric remodelling in normotensive subjects. J Hypertens 13:1818-1822

18. Frohlich ED, Apstein C, Chobanian AV et al (1992) The heart in hypertension. N Engl J Med 327:998-1008

19. De Boeck BW, Cramer MJ, Oh JK, van der Aa RP, Jaarsma W (2003) Spectral pulsed tissue Doppler imaging in diastole: a tool to increase our insight in and assessment of diastolic relaxation of the left ventricle. Am Heart J 146:411-419

20. Kasner M, Westermann D, Steendijk P et al (2007) Utility of Doppler echocardiography and tissue Doppler imaging in the estimation of diastolic function in heart failure with normal ejection fraction: a comparative Doppler-conductance catheterization study. Circulation 116:637-647

21. SAS Institute (2009) Knowledge base/product documentation. Available from http://support.sas.com/documentation/onlinedoc/ 91pdf/index.html, accessed 1 June 2009

22. Bachorik PS, Ross JW (1995) National Cholesterol Education Program recommendations for measurement of low-density lipoprotein cholesterol: executive summary. The National Cholesterol Education Program Working Group on Lipoprotein Measurement. Clin Chem 41:1414-1420

23. McLaughlin T, Abbasi F, Cheal K, Chu J, Lamendola C, Reaven G (2003) Use of metabolic markers to identify overweight individuals who are insulin resistant. Ann Intern Med 139:802-809

24. Warnick GR, Wood PD (1995) National Cholesterol Education Program recommendations for measurement of high-density lipoprotein cholesterol: executive summary. The National Cholesterol Education Program Working Group on Lipoprotein Measurement. Clin Chem 41:1427-1433

25. Khouri SJ, Maly GT, Suh DD, Walsh TE (2004) A practical approach to the echocardiographic evaluation of diastolic function. J Am Soc Echocardiogr 17:290-297

26. Park HS, Naik SD, Aronow WS et al (2006) Differences of lateral and septal mitral annulus velocity by tissue Doppler imaging in the evaluation of left ventricular diastolic function. Am J Cardiol 98:970-972

27. Uusitupa M, Siitonen O, Pyorala K, Lansimies E (1985) Left ventricular function in newly diagnosed non-insulin-dependent (type 2) diabetics evaluated by systolic time intervals and echocardiography. Acta Med Scand 217:379-388

28. Hammond IW, Devereux RB, Alderman MH, Laragh JH (1988) Relation of blood pressure and body build to left ventricular mass in normotensive and hypertensive employed adults. J Am Coll Cardiol 12:996-1004

29. Messerli FH, Sundgaard-Riise K, Reisin ED et al (1983) Dimorphic cardiac adaptation to obesity and arterial hypertension. Ann Intern Med 99:757-761

30. Pascual M, Pascual DA, Soria F et al (2003) Effects of isolated obesity on systolic and diastolic left ventricular function. Heart 89:1152-1156

31. Palmieri V, Bella JN, Arnett DK et al (2001) Effect of type 2 diabetes mellitus on left ventricular geometry and systolic 
function in hypertensive subjects: Hypertension Genetic Epidemiology Network (HyperGEN) study. Circulation 103: 102-107

32. Mustonen JN, Uusitupa MI, Tahvanainen K et al (1988) Impaired left ventricular systolic function during exercise in middle-aged insulin-dependent and noninsulin-dependent diabetic subjects without clinically evident cardiovascular disease. Am J Cardiol 62:1273-1279

33. Kimball TR, Daniels SR, Khoury PR, Magnotti RA, Turner AM, Dolan LM (1994) Cardiovascular status in young patients with insulin-dependent diabetes mellitus. Circulation 90:357-361

34. Poirier P, Bogaty P, Garneau C, Marois L, Dumesnil JG (2001) Diastolic dysfunction in normotensive men with well-controlled type 2 diabetes: importance of maneuvers in echocardiographic screening for preclinical diabetic cardiomyopathy. Diabetes Care 24:5-10

35. Tarumi N, Iwasaka T, Takahashi N et al (1993) Left ventricular diastolic filling properties in diabetic patients during isometric exercise. Cardiology 83:316-323

36. Chinali M, de Simone G, Roman MJ et al (2006) Impact of obesity on cardiac geometry and function in a population of adolescents: the Strong Heart Study. J Am Coll Cardiol 47:2267-2273

37. Sharpe JA, Naylor LH, Jones TW et al (2006) Impact of obesity on diastolic function in subjects $<$ or $=16$ years of age. Am J Cardiol 98:691-693

38. Whalley GA, Gusso S, Hofman P et al (2009) Structural and functional cardiac abnormalities in adolescent girls with poorly controlled type 2 diabetes. Diabetes Care 32:883-888 